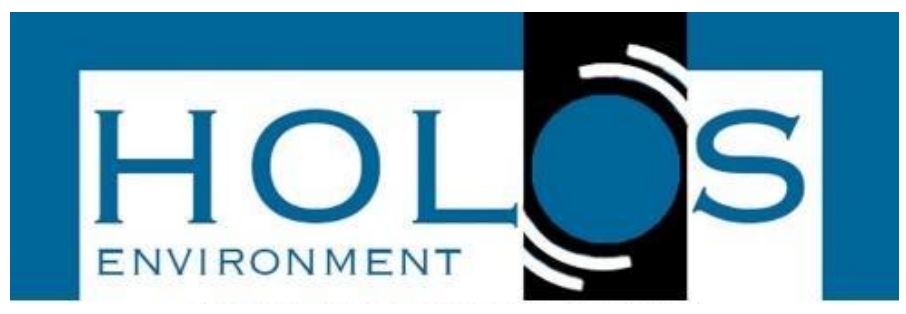

\title{
ANÁLISE DOS IMPACTOS AMBIENTAIS DECORRENTES DA DISPOSIÇÃO IRREGULAR DOS RESÍDUOS DE CONSTRUÇÃO E DEMOLIÇÃO NO BAIRRO ALTO BOM JESUS EM SERRA TALHADA -PE
}

\section{ANALYSIS OF ENVIRONMENTAL IMPACTS RESULTING FROM THE IRREGULAR DISPOSAL OF CONSTRUCTION AND DEMOLITION WASTE IN NEIGHBORHOOD ALTO BOM JESUS IN SERRA TALHADA-PE}

\author{
João Filipe Pereira de Lima1; Rafaella Pereira Marinho'; Elivelthon Carlos do \\ Nascimento ${ }^{1}$; Vitor Hugo de Oliveira Barros ${ }^{1}$
}

Artigo recebido em: 28/09/2021 e aceito para publicação em: 16/11/2021.

DOI: http://doi.org/10.14295/holos.v21i3.12454

\begin{abstract}
Resumo: No Brasil, a Construção Civil é um segmento de grande relevância para o crescimento econômico e social do Brasil. No entanto, é um dos setores que provocam impactos ambientais no país. Os Resíduos de Construção e Demolição (RCD) são materiais provenientes de construções, reformas, reparos e demolições de obras de construção civil, e os oriundos da preparação e da escavação de terrenos (CONAMA, 2002). Estes materiais, são encontrados, com frequência, descartados de maneira irregular em terrenos abandonados, gerando impactos ao meio ambiente. Na cidade de Serra Talhada, também foi possível encontrar em algumas regiões o descarte dos resíduos de construção e demolição de maneira irregular, o que provoca diversos problemas ambientais e sociais. Portanto, o presente estudo tem por principal objetivo o diagnóstico dos impactos ambientais causados pela deposição dos resíduos de construção e demolição no bairro do Alto Bom Jesus localizado no município de Serra Talhada-PE. Para isto, foram realizadas revisões bibliográficas sobre a temática, visitas técnicas ao bairro Alto Bom Jesus, com o objetivo de catalogar os impactos ambientais observados no local e a elaboração da Matriz de Impacto com a análise detalhada dos principais impactos ambientais observados na área. Dentre os impactos ambientais encontrados estão a contaminação química e biológica e a alteração das propriedades físicas do solo e da água; a alteração da qualidade do ar devido a presença de poeira ou gases; alteração nas condições de saúde e segurança da população devido ao aparecimento de vetores de doenças; alteração da qualidade paisagística; o aumento das despesas do município e os impactos sobre os arbustos, as árvores e os animais que podem estar localizados próximos aos locais de descarte de RCD.
\end{abstract}

Palavras-chave: Resíduo de construção e demolição. Impacto Ambiental. Matriz de Leopold.

\begin{abstract}
In Brazil, Civil Construction is a segment of great relevance for economic and social growth in Brazil. However, it is one of the sectors that cause environmental impacts in the country. Construction and Demolition Waste (CDW) are materials from constructions, renovations, repairs and demolitions of civil construction works, and those from the preparation and excavation of land (CONAMA, 2002). These materials are often found irregularly discarded in abandoned land, causing impacts to the environment. In the city of Serra Talhada, it was also possible to find in some regions the irregular disposal of construction and demolition
\end{abstract}

${ }^{1}$ Instituto Federal de Educação, Ciência e Tecnologia do Sertão Pernambucano (IF Sertão-PE), Petrolina, PE. E-mails: (joaodjona@gmail.com, rafaella.marinho@ifsertao-pe.edu.br, elivelthon.nascimento@ifsertaope.edu.br, vitor.barros@ifsertao-pe.edu.br) 
waste, which causes several environmental and social problems. Therefore, the main objective of this study is to diagnose the environmental impacts caused by the deposition of construction and demolition waste in the Alto Bom Jesus neighborhood located in the municipality of Serra Talhada-PE. For this, bibliographical reviews were carried out on the subject, technical visits to the Alto Bom Jesus neighborhood, with the objective of cataloging the environmental impacts observed in the place and the elaboration of the Impact Matrix with a detailed analysis of the main environmental impacts observed in the area. Among the environmental impacts found are chemical and biological contamination and changes in the physical properties of soil and water; the change in air quality due to the presence of dust or gases; changes in the health and safety conditions of the population due to the appearance of disease vectors; change in landscape quality; increased municipality expenditures and impacts on shrubs, trees and animals that may be located near CDW disposal sites.

Keywords: Construction and demolition waste. Environmental impact. Leopold's matrix.

\section{INTRODUÇÃO}

A Construção Civil é um segmento de grande importância para o crescimento econômico e social do Brasil, entretanto, é um dos setores de maiores impactos ambientais no país, devido ao elevado consumo de recursos naturais, alteração das paisagens ou geração de resíduos (MARINHO, 2018).

A tecnologia construtiva normalmente aplicada no Brasil contribui para o desperdício na execução das novas edificações. Os resíduos gerados nestas construções correspondem a aproximadamente $50 \%$ da quantidade em peso de resíduos sólidos urbanos (RSU) coletados nas cidades que possuem mais que 500 mil habitantes, seja no Brasil ou em outros países (SILVA et al., 2017).

Segundo a ABRELPE (2020), a quantidade total de RCD que os municípios coletaram no ano de 2019 foi de aproximadamente 45 milhões de toneladas. Este valor trata-se apenas do RCD coletado pelos logradouros públicos.

No Brasil, a Política Nacional de Resíduos Sólidos (PNRS), a Lei Federal $\mathrm{n}^{\circ}$ 13.305/2010 e seu Decreto nำ 7.404/2010 (BRASIL, 2010a; 2010b) determinam as principais diretrizes para a gestão e gerenciamento dos resíduos sólidos. Segundo a PNRS, todos os municípios brasileiros devem estabelecer Planos Municipais de Gestão Integrada de Resíduos Sólidos (PMGIRS), que devam atender às resoluções do Conselho Nacional de Meio Ambiente (CONAMA) e às legislações federais, estaduais e municipais.

Os resíduos de construção e demolição (RCD) estão entre as classes de resíduos sólidos listadas no PNRS que apresentam elevadas taxas de geração, o que constitui um desafio para o poder público municipal prover o gerenciamento adequado. No entanto, a fim de reverter esta situação, foram criadas ações como a Resolução CONAMA no $307 / 2002$, onde se estabelecem diretrizes, critérios e procedimentos para a Gestão dos Resíduos da Construção Civil. Esta resolução define os Resíduos de Construção e 
Demolição (RCD) como materiais provenientes de construções, reformas, reparos e demolições de obras de construção civil, e os oriundos da preparação e da escavação de terrenos, tais como: tijolos, blocos cerâmicos, concreto em geral, solos, rochas, metais, resinas, colas, tintas, madeiras e compensados, forros, argamassa, gesso, telhas, pavimento asfáltico, vidros, plásticos, tubulações, fiação elétrica etc., comumente chamados de entulhos de obras, caliça ou metralha.

Apesar de serem vistos como materiais de baixa periculosidade, seu descarte de maneira irregular em terrenos abandonados gera impactos ao meio ambiente. Dentre os impactos ambientais, estão as inundações causadas pelo estreitamento das margens de rios, poluição das águas e solos, indução à deposição de outros tipos de rejeitos que são atrativos para vetores de doenças além da poluição visual (IKAU et al., 2016).

De acordo com o artigo $1^{\circ}$ da Resolução 001/86 do CONAMA o impacto ambiental é definido como:

Qualquer alteração das propriedades físicas, químicas e biológicas do meio ambiente, causada por qualquer forma de matéria ou energia resultante das atividades humanas que, direta ou indiretamente, afetam:

I - a saúde, a segurança e o bem-estar da população;

II - as atividades sociais e econômicas;

III - a biota;

IV - as condições estéticas e sanitárias do meio ambiente;

$\mathrm{V}$ - a qualidade dos recursos ambientais.

Uma das maneiras de analisar os impactos ambientais causados pela disposição irregular de RCD é através da Matriz de Leopold. Trata-se de um instrumento largamente utilizado e conhecido por seus resultados eficazes. Esta matriz relaciona em um eixo as ações que provocam o impacto ambiental e no outro as condições ambientais que podem ser afetadas, auxiliando na identificação de alternativas que podem minimizar os impactos (LEOPOLD et al., 1971).

A Matriz de Impacto ou Matriz de Causa x Efeito ou Matriz de Leopold busca associar os impactos de uma ação particular com as variações de características ambientais da sua área de influência. É um método que relaciona o impacto de cada ação com o fator ambiental em células definidas pela interseção entre linhas e colunas, onde as linhas 
podem representar a ação impactante e as colunas afetam os fatores ambientais (SILVA, 2013).

Na cidade de Serra Talhada, que está localizada a $415 \mathrm{~km}$ da capital pernambucana e possui uma população estimada de 86.915 pessoas, também foi possível encontrar em algumas regiões o descarte dos resíduos de construção e demolição de maneira irregular, o que provoca diversos problemas ambientais e sociais.

A grande ocorrência de descarte irregular de Resíduo de construção e demolição (RCD) e os impactos ambientais provocados por este descarte, no bairro Alto Bom Jesus em Serra Talhada -PE, mostra a extrema importância da correta gestão dos resíduos de construção e demolição. Com isso, este artigo tem como principal objetivo diagnosticar os impactos ambientais causados pela deposição dos resíduos de construção e demolição no bairro Alto Bom Jesus, localizado no município de Serra Talhada-PE e seus resultados servirão como base para elaboração de soluções que venham minimizar os impactos gerados ao meio ambiente neste bairro.

\section{MATERIAIS E MÉTODOS}

\subsection{Caracterização da Área}

O município de Serra Talhada está localizado a $415 \mathrm{~km}$ da capital pernambucana, na Mesorregião do Sertão Pernambucano, Microrregião do Pajeú, a uma altitude de 429 metros. Possui uma população estimada de 87.467 habitantes (IBGE, 2021). Trata-se da segunda cidade mais importante do Sertão de Pernambuco e o principal município da Mesorregião do Sertão Pernambucano.

Serra Talhada possui uma área total de $2.980,007 \mathrm{~km}^{2}$, composta por diversos bairros e distritos, dentre eles o Bairro Alto Bom Jesus (Figura 1), onde este estudo foi realizado.

O Bairro Alto Bom Jesus abrange grandes centros comerciais como o Assaí Atacadista e o Home Center Tupan. Trata-se de um bairro que está em processo de crescimento, com muitas residências em fase de construção. 
Figura 1- Bairro Alto Bom Jesus

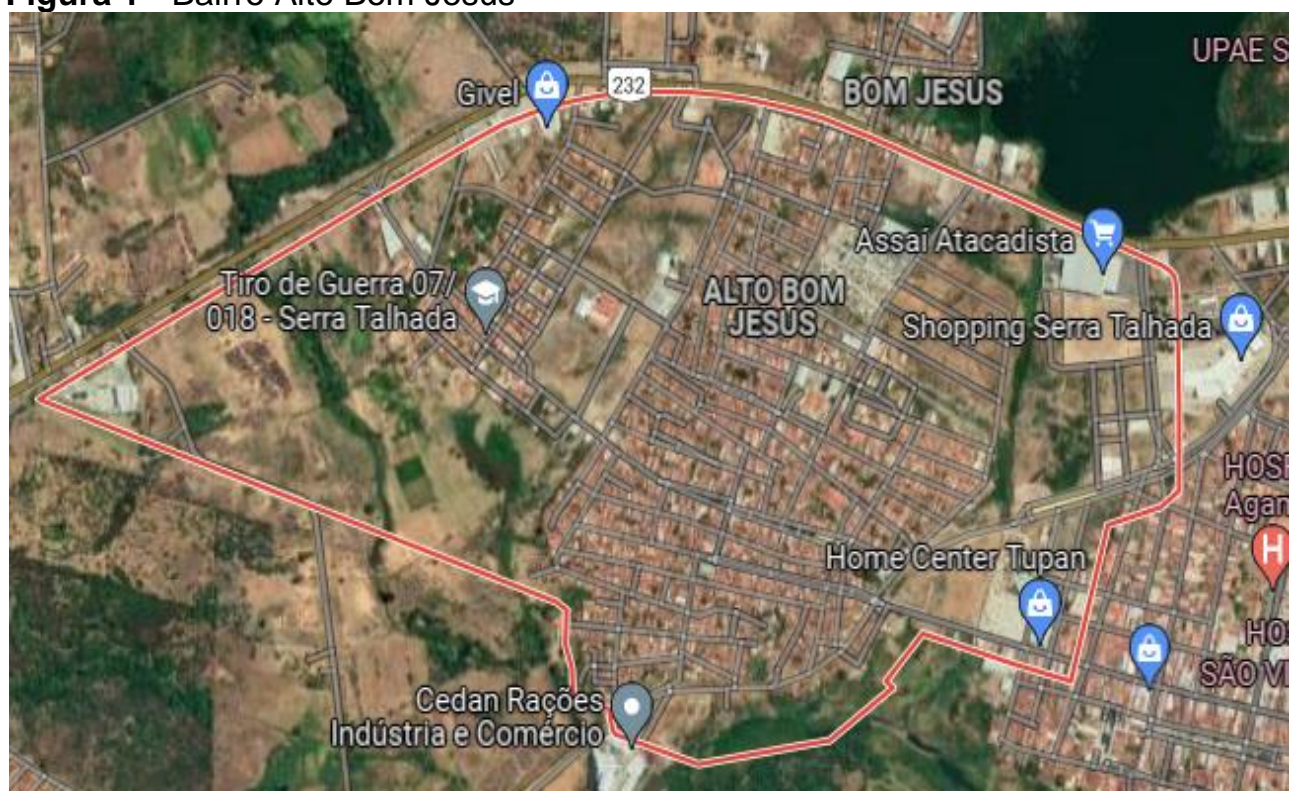

Fonte: Google Earth (2021)

\subsection{Procedimentos metodológicos}

O trabalho se desenvolveu em quatro etapas, conforme fluxograma apresentado na Figura 2. A primeira etapa consistiu na elaboração de uma revisão bibliográfica através da pesquisa de artigos científicos e periódicos relacionados ao tema.

Figura 2 - Fluxograma das etapas para realização da pesquisa

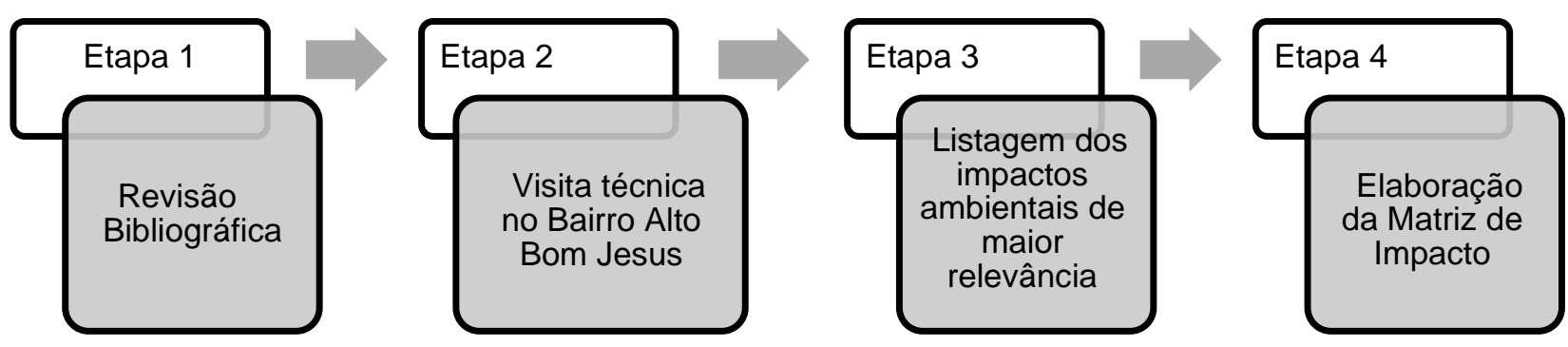

Fonte: Autores

A segunda etapa se deu através da realização de uma visita técnica no bairro Alto Bom Jesus, do município de Serra Talhada, com o objetivo de catalogar os impactos ambientais observados no local por meio de registros fotográficos.

$\mathrm{Na}$ terceira etapa, foi realizada a listagem dos impactos ambientais de maior relevância. 
A última etapa consistiu na elaboração da Matriz de Impacto proposta por Santos (2014), com a análise detalhada dos principais impactos ambientais observados na área, em função de aspectos da ocupação.

\subsubsection{Avaliação dos impactos ambientais com a Matriz de Leopold}

A avaliação de impactos ambientais iniciou-se na etapa 2 através da visita técnica no Bairro Alto Bom Jesus, onde foram catalogados os impactos ambientais observados no local por meio de registros fotográficos.

Aos impactos ambientais identificados foram atribuídas pontuações conforme apresentado na Tabela $1 \mathrm{e}$, através da Equação 1, obteve-se a quantificação do impacto total, cuja pontuações variam de -12 (pior caso) a +12 (melhor caso). Com base nestes resultados foi montada a Matriz de Leopold dos impactos ambientais provocados pela deposição irregular do RCD do Bairro Alto Bom Jesus.

Tabela 1 - Pontuação por critério

\begin{tabular}{|c|c|c|c|}
\hline Caráter (Ca) & Negativo $=-1$ & Neutro $=0$ & Positivo= 1 \\
\hline Importância (I) & Alto $=3$ & Médio= 2 & Baixo= 1 \\
\hline Cobertura (Co) & Regional= 3 & Local= 2 & Pontual= 1 \\
\hline Duração (D) & $\begin{array}{l}\text { Permanente }= \\
3\end{array}$ & Médio= 2 & Curta $=1$ \\
\hline Reversibilidade (C) & Irreversível= 3 & Parcial $=2$ & Reversível= 1 \\
\hline
\end{tabular}

$\operatorname{IT}($ Impacto Total $)=C a \times(I+C o+D+R)$

\section{RESULTADOS E DISCUSSÃO}

\subsection{Impactos ambientais no Bairro Alto Bom Jesus}

A partir da visita técnica foi possível observar que o Bairro Alto Bom Jesus, por se tratar de um local em processo de crescimento, com muitas residências em fase de construção ou passando por reformas, se tornou uma área muito suscetível à acumulação de RCD. 
No Bairro Alto Bom Jesus foram identificados 19 pontos de deposição irregular de resíduos de construção e demolição (RCD) encontrados das seguintes formas:

- Depositados nos logradouros em frente ou próximos ao local das obras (Figuras 3a e 3b);

- Depositados em terrenos para serem possivelmente utilizados como aterros (Figura 3c);

- Descartados em terrenos baldios (Figura 3d).

Figura 3 - a) e b) RCD depositados nos logradouros do Bairro Alto Bom Jesus, c) RCD depositados em terrenos para serem possivelmente utilizados como aterro e d) RCD descartados em terrenos baldios
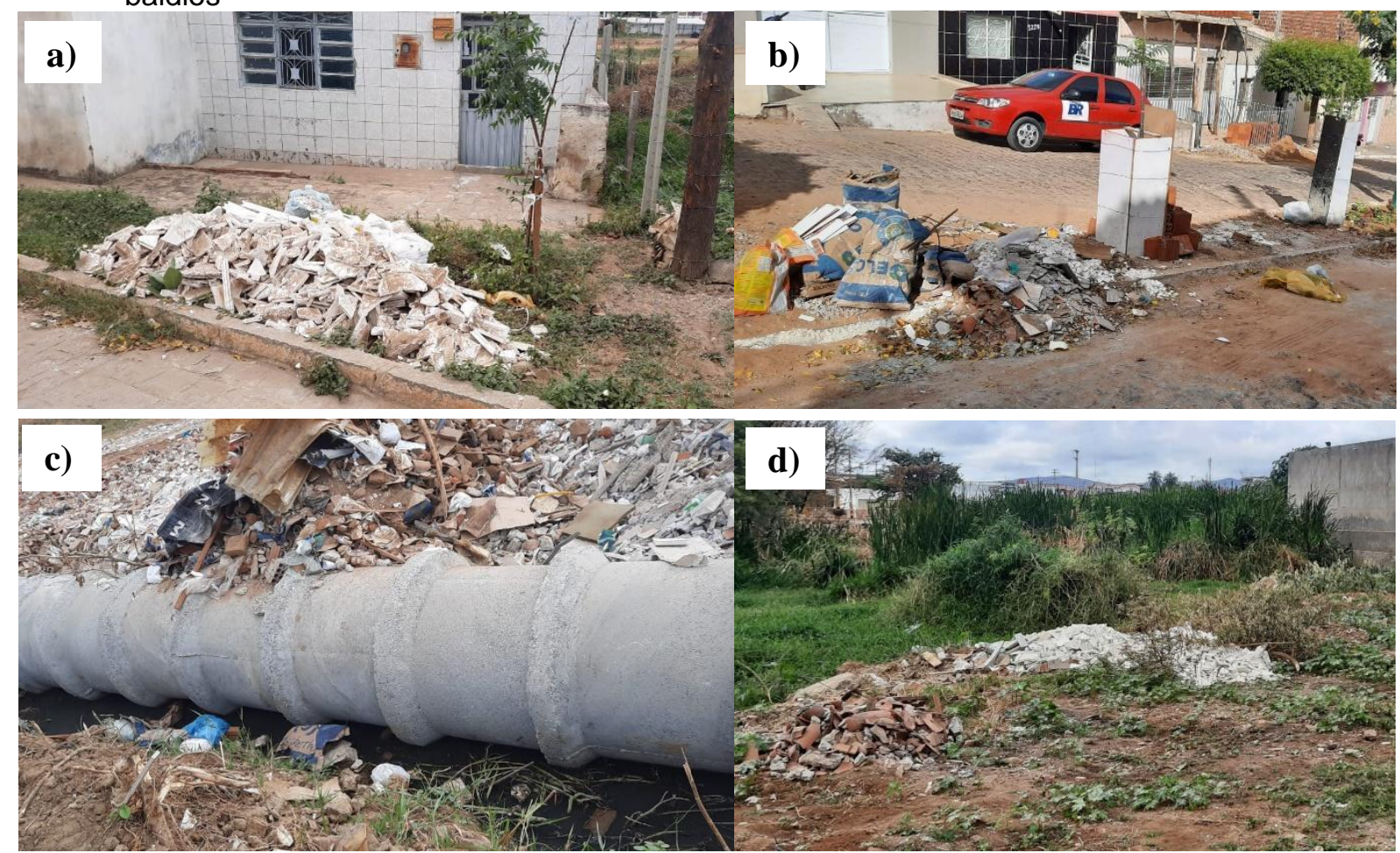

Fonte: Autores

Além do RCD estas áreas são alvos frequentes de descarte de RSU, aumentando os prejuízos à saúde pública e ao saneamento ambiental da cidade, devido ao aparecimento de vetores de doenças, conforme apresenta Figura 4. 
Figura 4 - RSU no mesmo local do RCD
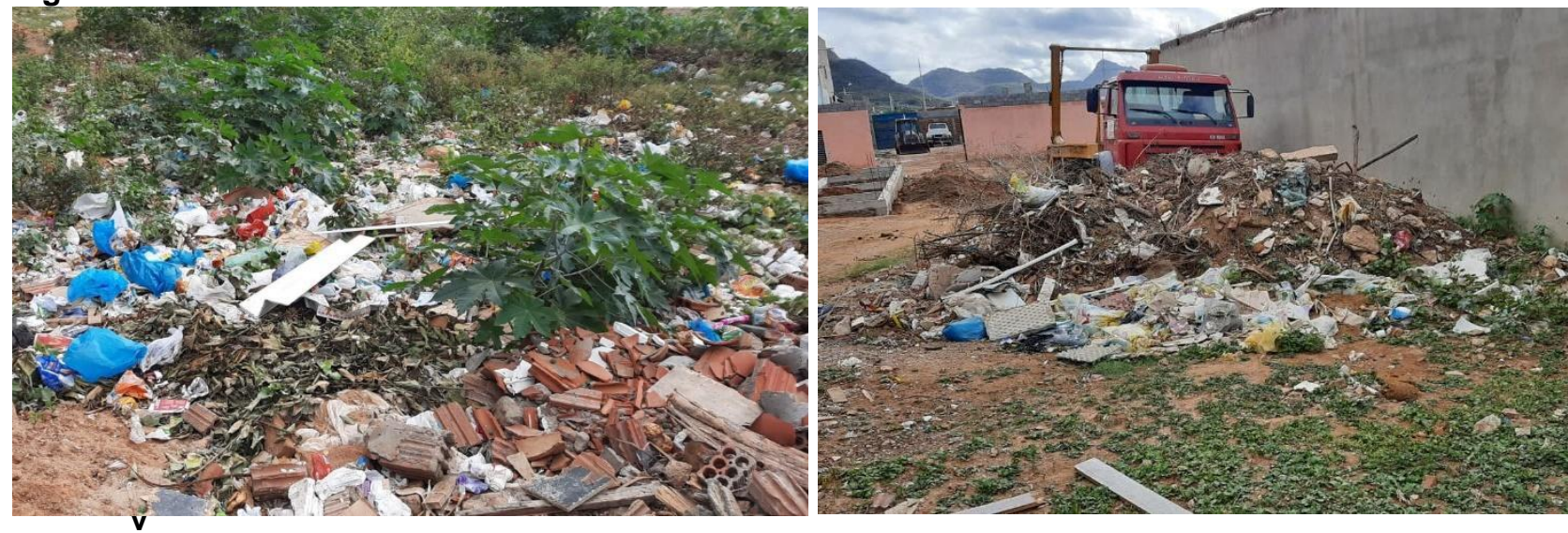

Fonte: Autores

Outro problema observado foi o lançamento de esgoto domiciliar no solo, devido à falta de infraestrutura para captação e destinação adequada no mesmo local onde também é descartado RCD, ver Figura 5.

Figura 5 - Lançamento de esgoto domiciliar e RCD no solo

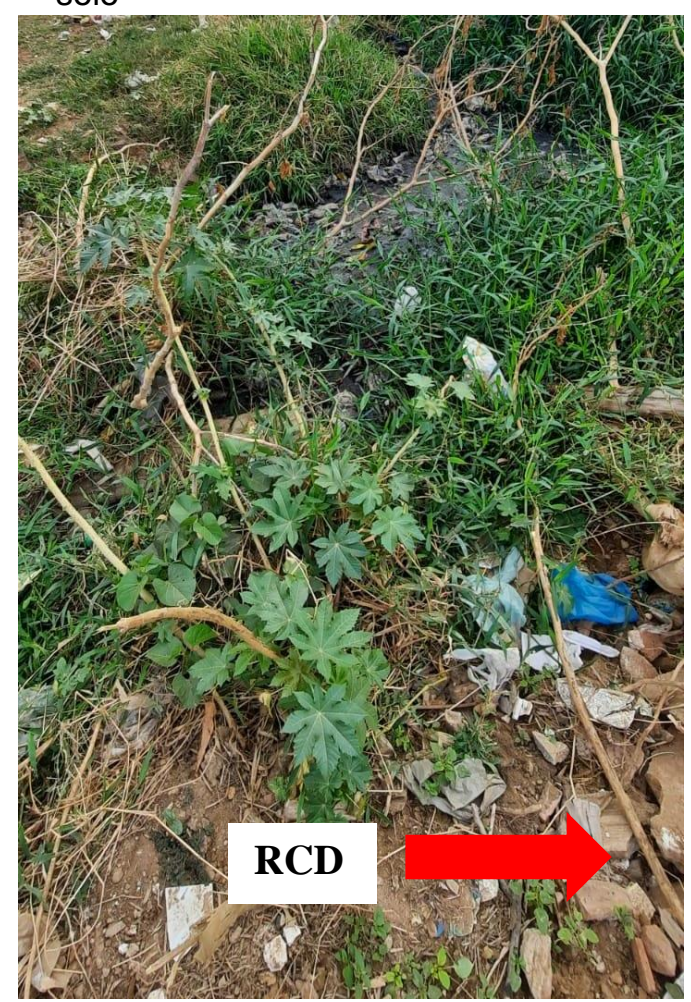

Fonte: Autores

O lançamento do esgoto domiciliar na superfície do terreno, além de contaminar o solo, pode contaminar o Rio Pajeú, especialmente em períodos chuvosos, por estar próximo de sua localização. 
Diante disso, foi possível observar que os principais efeitos dos impactos sociais provocados pela deposição de RCD de forma irregular estão ligados à saúde e segurança devido ao aparecimento de vetores de doenças, alteração da qualidade paisagística e o aumento das despesas do município. Quanto aos impactos físicos, no solo e na água podem ocorrer contaminação química e biológica e alteração de suas propriedades físicas; enquanto $o$ ar pode ter sua qualidade alterada devido a presença de poeira ou gases. Já os efeitos dos impactos biológicos são sobre os arbustos, árvores e animais que podem estar localizados próximos aos locais de descarte de RCD.

\subsection{Análise dos impactos ambientais no Bairro Alto do Bom Jesus}

Os impactos ambientais ocasionados pela deposição irregular de RCD no Bairro Alto Bom Jesus foram quantificados de acordo com o Caráter (Ca), a Importância (I), a Cobertura (Co) e a Reversibilidade (C). Através da pontuação de cada parâmetro, calculouse o impacto total e foi montada a Matriz de Leopold proposta por Santos (2014), conforme apresenta a Tabela 2. Para os aspectos de geração de resíduos de construção e demolição, alteração da cobertura vegetal do solo e contaminação do Rio Pajeú foram registrados valores que variaram entre 0 (zero) e -10 (dez), não apresentando notas positivas.

Como se obteve apenas aspectos negativos, não foi realizado o balanceamento dos aspectos positivos com os negativos, gerando um resultado final igual a pontuação negativa, tendo valor de - 332 (menos trezentos e trinta e dois). Este resultado expressa o quanto as condições atuais impactam no meio ambiente do Bairro Alto Bom Jesus, gerando efeitos na qualidade de vida daqueles que nele habitam. 
Tabela 2 - Matriz de Leopold - Impactos Ambientais ocasionados deposição irregular do RCD no bairro Alto Bom Jesus

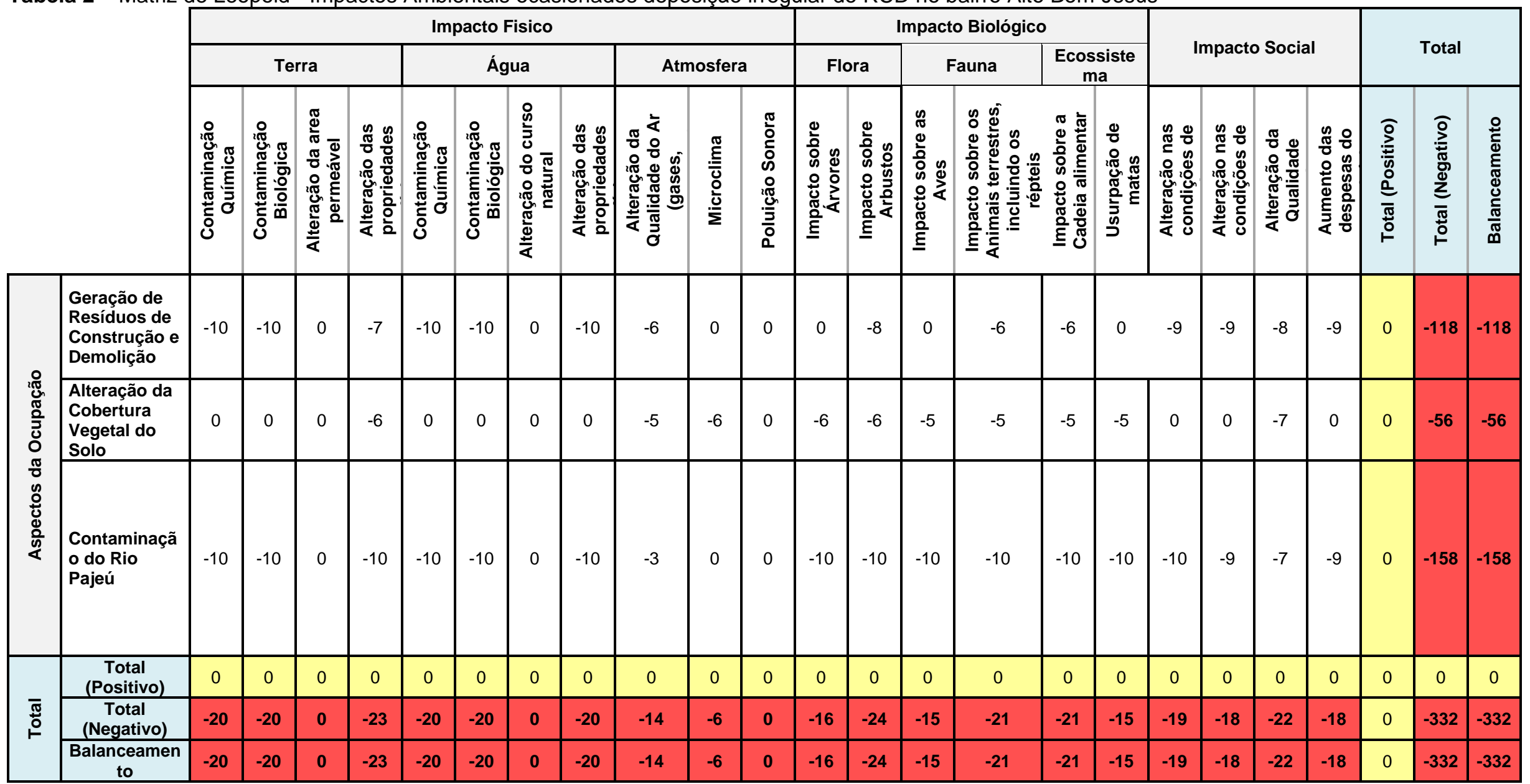

Fonte: Autores 


\section{CONCLUSÕES}

A deposição irregular de Resíduos de Construção e Demolição gera impactos ambientais, físicos, biológicos e sociais. Dentre os impactos ambientais encontrados estão a contaminação química e biológica e a alteração das propriedades físicas do solo e da água; a alteração da qualidade do ar devido a presença de poeira ou gases; alteração nas condições de saúde e segurança da população devido ao aparecimento de vetores de doenças; alteração da qualidade paisagística; o aumento das despesas do município e os impactos sobre os arbustos, as árvores e os animais que podem estar localizados próximos aos locais de descarte de RCD.

Diante dos impactos identificados, percebe-se que a situação se agrava nos períodos chuvosos, aumentando as chances de contaminação do Rio Pajeú e de proliferação de doenças para a população. É prática comum dos moradores utilizarem os resíduos da construção como aterro em suas casas para evitar isso.

Para minimizar tais impactos, é necessário que sejam executados o saneamento básico e drenagem e infraestrutura no bairro, a fim de evitar a contaminação do solo e da água do Rio Pajeú, além de ampliar, incentivar e propor o reaproveitamento do RCD.

\section{REFERÊNCIAS}

ABRELPE - Associação Brasileira de Empresas de Limpeza Pública e Resíduos Especiais: Panorama dos resíduos sólidos no Brasil 2020. São Paulo. Disponível em: https://abrelpe.org.br/panorama-2020// Acesso em: 20 set. 2021.

BRASIL. Ministério do Meio Ambiente. Resolução no 307, de 5 de julho de 2002. Estabelece diretrizes, critérios e procedimentos para a gestão dos resíduos da construção civil. Diário Oficial [da] República Federativa do Brasil, Conselho Nacional do Meio Ambiente, Brasília, 17 jul. 2002, Seção 1, p. 95-96.

BRASIL. Ministério do Meio Ambiente. Resolução no 001, de 23 de janeiro de 1986. Dispõe sobre critérios básicos e diretrizes gerais para a avaliação de impacto ambiental. Diário Oficial [da] República Federativa do Brasil, Conselho Nacional do Meio Ambiente, Brasília, de 17 de fevereiro de 1986, Seção 1, páginas 2548-2549.

BRASIL. Lei no 12.305, de 2 de agosto de 2010a. Institui a Política Nacional de Resíduos Sólidos; altera a Lei no 9.605, de 12 de fevereiro de 1998; e dá outras providênci as. Diário Oficial [da] República Federativa do Brasil, Brasília, 3 ago. 2010, Seção 1, p. 3. 
BRASIL. Decreto no. 7.404, de 23 de dezembro de 2010b. Regulamenta a Lei o․ 12.305, de 2 de agosto de 2010, que institui a Política Nacional de Resíduos Sólidos, cria o Comitê Interministerial da Política Nacional de Resíduos Sólidos e o Comitê Orientador para a Implantação dos Sistemas de Logística Reversa, e dá outras providências. Diário Oficial [da] República Federativa do Brasil, Brasília.

IBGE - Instituto Brasileiro de Geografia e Estatística (2021). Estimativa Populacional. Rio de Janeiro. Disponível em: https://www.ibge.gov.br/cidades-e-estados/pe/serratalhada.html. Acesso em: 20 set. 2021.

IKAU, R; JOSEPH, C, TAWIE, R. Factors influencing waste generation in the construction industry in Malaysia. Procedia - Social and Behavioral Sciences, v. 234, p. 11-18, 2016 https://doi.org/10.1016/j.sbspro.2016.10.213

LEOPOLD, L.B A procedure for evaluating environmental impacts. Geol. Surv. Circ, v. 645, 1971. https://doi.org/10.3133/cir645

MARINHO, R. P. (2018). Melhoramento de solo colapsível e expansivo de Santa Maria da Boa Vista-PE. Dissertação (Mestrado) - Universidade Federal de Pernambuco. Recife-PE, $114 \mathrm{f}$.

SANTOS, Daris Correia dos. Atuação da defesa civil na avaliação e análise de riscos ambientais. Revista Eletronica em Gestão, Educação e Tecnologia Ambiental REGET , v. 18 n. 1, p. 14 - 24, abr. 2014. https://doi.org/10.5902/2236117010015

SILVA, M. L. O. Impactos ambientais e sociais decorrentes de ocupações nas margens do Rio Capibaribe, Município de São Lourenço da Mata /PE. 2013. 114 f. Monografia (Especialização) - Curso de Gestão e Controle Ambiental, Programa de Pós Graduação em Gestão e Controle Ambiental, Universidade de Pernambuco, Recife, 2013

SILVA, L. R. M.; MATOS, E. T. A. R.; FISCILETTI, R. M. D. S. Resíduo sólido ontem e hoje: evolução histórica dos resíduos sólidos na legislação ambiental brasileira. Revista AREL FAAR. Amazon's Research na Environmental Law, v. 5, n. 2, p. 126-142, 2017. 